\title{
Métodos Numéricos de Volumes Finitos aplicados em Hidrodinâmica Ideal via Octave
}

\author{
Raphael de O. Garcia* $\quad$ Samuel R. de Oliveira \\ Departamento de Matemática Aplicada, IMECC, Unicamp, \\ 13083-859, Campinas, SP \\ E-mail: gr.gubim@gmail.com, samuel@ime.unicamp.br,
}

\section{$\underline{\text { RESUMO }}$}

Nas últimas décadas, Métodos Numéricos de Volumes Finitos vêm sendo desenvolvidos, aprimorados e aplicados em sistemas de equações diferenciais parciais (EDP's) hiperbólicos não lineares dependentes do tempo [4]. As leis de conservações são escritas por sistemas de EDP's e a modelagem da maioria dos problemas em Ciências e/ou Engenharias parte de tais leis.

Em particular, um sistema que se destaca é o formado pelas equações de Euler que modelam o escoamento de um fluido por possuir dificuldades e desafios referentes à obtenção de soluções numéricas [3]. O caráter puramente não linear dessas equações possibilitam a formação dos três tipos de ondas elementares: ondas de choque, ondas de rarefação e ondas de contato, que aparecem como descontinuidades na solução das equações de Euler [6].

No que diz respeito a Métodos Numéricos, cada um possui suas próprias propriedades que influenciam diretamente na solução numérica tornando-os adequados ou não dependendo da aplicação em questão.

Neste trabalho são feitas comparações entre os métodos de volumes finitos aplicados às equações de Euler Unidimensionais, dada pelas seguintes expressões:

$$
\begin{array}{ll}
\frac{\partial}{\partial t} \rho+\frac{\partial}{\partial x}(\rho v)=0 & \text { (equação da continuidade) } \\
\frac{\partial}{\partial t} \rho v+\frac{\partial}{\partial x}\left(\rho v^{2}+p\right)=0 & \text { (equação do movimento) } \\
\frac{\partial}{\partial t} E+\frac{\partial}{\partial x}(v(E+p))=0 & \text { (equação da energia) }
\end{array}
$$

em que

$$
\begin{array}{ll}
\rho=\rho(x, t) & \text { (densidade do fluido em escoamento) } \\
v=v(x, t) & \text { (velocidade do fluido) } \\
p=p(x, t) & \text { (distribuição de pressão no fluido) } \\
E=E(x, t) & \text { (energia total do fluido) } \\
\epsilon=\epsilon(x, t) & \text { (energia interna do fluido) } \\
\gamma & \text { (calor específico do fluido) }
\end{array}
$$

com

$$
E=\frac{1}{2}\left(\rho v^{2}+\epsilon\right) \quad \text { e } \epsilon=\frac{p}{(\gamma-1) \rho} .
$$

\footnotetext{
${ }^{*}$ Professor do Instituto Federal de Educação, Ciência e Tecnologia de Goiás - Câmpus Jataí
} 
Os métodos numéricos Lax-Friedrichs, Nessyahu-Tadmor [5], Lax-Wendroff, Godunov, esquemas essencialmente não oscilatórios ponderados (WENO) com Runge-Kutta [2] e Runge-Kutta com LaxWendroff [1] foram implementados em Octave e comparados com relação ao tempo gasto de CPU, consumo de memória, quantidade de operações, oscilações e/ou dissipações numéricas, ordem de precisão e estabilidade. Assim, têm-se tabelas de comparações entre métodos que podem auxiliar na escolha de métodos numéricos para problemas similares. Além disso, gráficos comparativos das soluções aproximadas do sistema (1) foram elaborados com o mesmo intuito.

Consequentemente, o trabalho explorou o uso do Octave - ambiente Linux/GNU - em questões de análise numérica relevantes ao uso de métodos numéricos de volumes finitos aplicados na obtenção de soluções aproximadas para as equações de Euler (hidrodinâmica ideal).

Palavras-chave: Métodos de Volumes Finitos, Equações de Euler, Octave

\section{Referências}

[1] W. Hundsdorfer, J. G. Verwer, "Numerical Solution of Time-Dependent Advection-DiffusionRection Equations", Springer, New York, 2003.

[2] G. -S. Jiang, C. -W. Shu, Efficient Implementation of Weighted ENO Schemes, Journal of Computational Physics, 126 (1996) 202-228.

[3] R. J. Leveque, "Finite Volume Methods for Hyberbolic Problems", Cambridge University Press, United States of America, 2006.

[4] J. D. Logan, "An Introduction to Nonlinear Partial Differential Equations", John Wiley \& Sons, New Jersey, 2008.

[5] H. Nessyahu, E. Tadmor, Non-Oscillatory Central Differencing for Hyperbolic Conservation Laws, Journal Computational Physics, 87, n. 2 (1990) 408-463.

[6] E. F. Toro, "Riemann Solvers and Numerical Methods for Fluid Dynamics - A Practical Introduction", Springer, Germany, 1999. 